\title{
Standardising measurement of tumour vascularity by imaging: recommendations for ultrasound, computed tomography, magnetic resonance imaging and positron emission tomography
}

\author{
Adrian K. Dixon • Fiona J. Gilbert \\ Received: 27 January 2012 / Accepted: 4 February 2012 / Published online: 29 April 2012 \\ (C) European Society of Radiology 2012
}

\begin{abstract}
This review analyses the need for, and likely impact of, four subsequent papers which discuss the importance of standardisation of ultrasound (US), computed tomography (CT), magnetic resonance imaging (MRI) and positron emission tomography (PET) when assessing tumour vascularity. This is particularly important when measuring the vascular effects of therapeutic agents in oncological research and practice. As imaging inexorably moves from the subjective interpretative art-form of the past into its modern role as a fully fledged objective scientific discipline, it is incumbent on all radiologists to understand the need for strict adherence to perceived best practice when evaluating lesions as part of trials. Indeed trials may only be funded by pharmaceutical companies and other grant-giving bodies if rigorous adherence to imaging protocols and quality assurance is in place.

Key Points

- Various imaging methods can now robustly assess tumour vascular support.

- US, CT, MRI and PET are increasingly used to assess tumour vascularity.

- These techniques have reached technical maturity for use in therapeutic oncological trials.

- Consensus guidelines about using these techniques in assessing tumour vascularity are introduced.

- Image acquisition protocols and quality assurance must be established for large trials.
\end{abstract}

A. K. Dixon $(\varangle) \cdot$ F. J. Gilbert

Department of Radiology, Addenbrooke's Hospital and the

University of Cambridge, Cambridge University Hospitals NHS

Foundation Trust,

Level 5, Box 218, Hills Road,

Cambridge CB2 0QQ, UK

e-mail: Adrian.dixon@european-radiology.org
Keywords Tumour vascularity · Angiogenesis · Early phase trials $\cdot$ Perfusion quantification $\cdot$ Response assessment

This issue embraces four seminal articles [1-4] reviewing how functional information can be accurately, repeatedly and noninvasively collected from cross-sectional imaging techniquesusing ultrasound (US), computed tomography (CT), magnetic resonance imaging (MRI) and positron emission tomography (PET). This information can be used to assess factors related to the vascularity of a tumour and provide prognostic information and also the likely response to various therapeutic agents. Such prediction of response is of crucial importance when the therapeutic agent is costly and carries serious side effects, such as many oncological drugs. As change in vascularity may be a genuine indication of response to treatment, together with associated alterations in glucose metabolism, these new radiological techniques become of paramount importance. Indeed, the tumour micro-environment is now an accepted biomedical marker [5] and it is important that radiological techniques are used to the full in order to assess such aspects. Thus, oncologists, health administrators and pharmaceutical companies now view such functional studies as an extremely important tool in their search for the optimal and most cost-effective therapy that can improve the health of the patient with the minimum of side effects. In this era of stratified medicine the importance of phenotyping the patient becomes increasingly relevant in order to select the correct combination of therapies.

Another reason for the need for these papers is that pharmacological companies demand robust, repeatable, reproducible and reliable measures, which are readily available to replace survival as the outcome measure. For the majority of tumours the wait for survival information would take many years. The 
RECIST recommendations have ensured that radiological size change is used in a reliable manner, but size change can take many months to appear. The advantage of early surrogate endpoints can result in fast-track assessments of novel drugs in development with stop/go decisions taken by pharmaceutical companies at a much earlier stage. However, all such new measures require excellent quality assurance and reproducibility with evidence of validation and qualification which will satisfy the FDA and other regulatory bodies. For example, in the UK, such evidence would be necessary for NIHR and other grants; in America the NIH would require similar proof of robustness and the Quantitative Imaging Biomarker Alliance (QIBA) has been set up to do this across a number of techniques (http://qibawiki.rsna.org/index.php?title=Main_Page); the EIBIR is leading on such initiatives in Europe.

It was Lord Kelvin who suggested that if something cannot be measured, it is not science-or words to that effect. The jury is out on which of the following quotes he actually said: "When you can measure what you are speaking about, and express it in numbers, you know something about it...", "If you cannot measure it, you cannot improve it" or "To measure is to know". Maybe he said them all at different times. Applied to Radiology, his principle is that as a profession we were for too long concerned with subjective interpretation and not sufficiently concerned with objective measurements. In the UK, there were several notable exceptions. George Simon long ago advocated measuring structures on a chest radiograph [6]. Nuclear medicine experts have long been able to measure renal function and iodine uptake in the thyroid. Hounsfield's breakthrough with CT allowed measurements of tissue attenuation [7]. Ian Isherwood and colleagues in Manchester subsequently exploited CT attenuation and subtle differences therein to the full [8]. Ken Miles then revolutionised CT measurements by applying nuclear medicine principles to provide CT perfusion data [9]. Dynamic enhancement of the breast was a natural progression [10]; multicentre trials illustrated the effort required to maintain image quality and uniformity across numerous imaging departments [11].

Only slowly, however, did these techniques capture the imagination of the radiology equipment manufacturers. Frequently the various measurements were made by in-house software and add-on hardware. Indeed, it took some time before such data could be directly measured on the main consoles and workstations - and even then at quite a price!

Scientists then realised that measurements made using one technique may not provide quite the same information as those collected by another. Of course it is appreciated that perfusion information is simpler to obtain using CT than MRI by virtue of the precise linear relationship of $\mathrm{CT}$ attenuation to iodine concentration. In MRI, the relationship between gadolinium concentration and signal intensity is much more complex and highly sequence-specific. Functional data from US and PET require detailed knowledge of physics, etc. It then became apparent that, even using the same technique, it was not a given that a relatively robust assessment (e.g. CT perfusion) measured in one institution would necessarily be the same as that measured at another. Differences in CT hardware/ software, CT protocols including methodology of contrast medium administration, the software used to assess perfusion and, finally, the human input required to obtain parameters such as regions of interest all contributed to variations in these crucial measurements [12]. As these measurements have become increasingly important in the design of pharmaceutical trials and indeed the management of patients, it has become imperative to ensure accuracy in every stage of such objective measurements. Hence the interest in variation within and between readers and between institutions. And hence the need for groups of scientists to collaborate and produce guidelines to ensure that a common approach is used for all such functional assessments. These concerns led to the following four papers describing a uniform approach for these measurements in US, CT, MRI and PET. The papers emanated from proceedings of a workshop attended by scientists belonging to an International Imaging Network of Experimental Cancer Medicine Centres (ECMC): imaging experts and oncologists met up and established various consensus statements using a modified Delphi approach.

The first paper by Leen et al. [1], "Dynamic contrast enhanced ultrasound assessment of the vascular effects of novel therapeutics in early stage trials", concludes that dynamic contrast enhanced (DCE) US is safe, well tolerated, available at the bedside and reproducible. US contrast agents provide blood pool information that may complement outputs from other imaging techniques. It is advised that DCE-US should be used in conjunction with other methods in future clinical trials to compare their reproducibility and predictive efficacy.

The second paper by Miles et al. [2], "Current status and guidelines for the assessment of tumour vascular support with dynamic contrast enhanced-computed tomography", provides reassurance that DCE-CT has reached a technical maturity, allowing its use for the assessment of tumour vascular support in therapeutic trials. Nevertheless, this first set of consensus guidelines should further aid the application of DCE-CT to the evaluation of tumour vascular physiology.

The third paper, by Leach et al. [3], is perhaps the most complex: "Evaluating vascular function for early stage clinical trials using dynamic contrast enhanced magnetic resonance imaging". It concludes by stating that recent technological advances in MRI provide interesting physiological information in a wide range of clinical and experimental situations. However, these advances necessitate careful quality assurance and standardisation of methods within and between trials if scientific progress is to be made. The paper provides recommendations for standardising approaches within a trial and for developing more detailed quality assurance standards. 
The final paper is by Aboagye et al. [4]: "Recommendations for measurement of tumour vascularity with positron emission tomography in early phase clinical trials". The paper concludes that ${ }^{15} \mathrm{O}$-water measurements represent the "gold standard" for assessing blood flow/tissue. However, the early phase of ${ }^{18} \mathrm{~F}$ FDG uptake curve may also become useful to estimate perfusion, and recent developments with new radiopharmaceuticals may well provide robust measures of angiogenesis growth factors. Once again, it is important to understand and implement techniques to ensure that the measurements are reproducible. This article recommends strategies for assuring imaging equipment stability, radiotracer specifications, dosimetry considerations, region-of-interest definition and quantifications, and reporting of PET data in order to enhance the use of these technologies within the wider cancer community.

These four papers should become regarded as benchmark papers for future pre-clinical and clinical trials evaluating bioimaging markers of tumours before, during and after therapeutic intervention using the exciting opportunities offered by modern imaging techniques.

\section{References}

1. Leen EL, Averkiou MA, Arditi M et al (2012) Dynamic contrast enhanced ultrasound (DCE-US) assessment of the vascular effects of novel therapeutics in early stage trials. Eur Radiol 22
2. Miles, KA, Lee T-Y, Goh V et al (2012) Current status and guidelines for the assessment of tumour vascular support with dynamic contrast enhanced-computed tomography. Eur Radiol 22

3. Leach MO, Morgan B, Tofts PS et al (2012) Evaluating vascular function for early stage clinical trials using dynamic contrast enhanced magnetic resonance imaging. Eur Radiol 22

4. Aboagye EO, Gilbert FJ, Fleming I et al. (2012) Recommendations for measurement of tumour vascularity with positron emission tomography in early phase clinical trials. Eur Radiol 22

5. Hanahan D, Weinberg RA (2011) Hallmarks of cancer: the next generation. Cell 144:646-674

6. Simon G (1962) Principles of chest X-ray diagnosis, 2nd edn. Butterworths, London

7. Hounsfield GN (1973) Computerized transverse axial scanning (tomography). 1. Description of system. Br J Radiol 46:10161022

8. Adams JE, Chen SZ, Adams PH, Isherwood I (1982) Measurement of trabecular bone mineral by dual energy computed tomography. $\mathrm{J}$ Comput Assist Tomogr 6:601-607

9. Miles KA, Hayball M, Dixon AK (1991) Colour perfusion imaging: a new application of computed tomography. Lancet 337:643-645

10. Heywang SH, Wolf A, Pruss E, Hilbertz T, Eiermann W, Permanetter W (1989) MR imaging of the breast with GdDTPA: use and limitations. Radiology 171:95-103

11. Leach MO, Boggis CR, Dixon AK, MARIBS study group et al (2005) Screening with magnetic resonance imaging and mammography of a UK population at high familial risk of breast cancer: a prospective multicentre cohort study (MARIBS). Lancet 365:1769-1778

12. Goh V, Shastry M, Engledow A et al (2011) Commercial software upgrades may significantly alter Perfusion CT parameter values in colorectal cancer. Eur Radiol 21:744-749 